\title{
Downregulation of miR-142-5p promotes tumor metastasis through directly regulating CYR61 expression in gastric cancer
}

\author{
Jing Yan ${ }^{1} \cdot$ Bing Yang ${ }^{1} \cdot$ Shuye Lin $^{1} \cdot$ Rui Xing ${ }^{1} \cdot$ Youyong Lu ${ }^{1}$
}

Received: 22 April 2018 / Accepted: 18 August 2018 / Published online: 3 September 2018

(c) The International Gastric Cancer Association and The Japanese Gastric Cancer Association 2018

\begin{abstract}
Background Recurrence is a primary cause of gastric cancer (GC)-related deaths. We reported previously that low expression of miR-142-5p could predict recurrence in GC. The present study aimed to investigate the function and mechanism of miR-142-5p in metastasis of GC.

Methods MiR-142-5p expression was detected in 101 GC samples by qRT-PCR. Its clinical significance was statistically analyzed. The roles of miR-142-5p and its candidate target gene CYR61 in metastasis were determined both in vivo and in vitro. Results MiR-142-5p downregulation was significantly associated with the recurrence $(P=0.031)$ and poor prognosis of GC $(P=0.043)$. MiR-142-5p inhibited cancer cell migration and invasion both in vitro and in vivo. CYR61 was identified as a novel direct target of miR-142-5p by bioinformatics analysis of target prediction and luciferase reporter assay. The re-expression and knockdown of CYR61 could, respectively, rescue the effects induced by miR-142-5p overexpression and knockdown. MiR-142-5p attenuated GC cell migration and invasion, at least partially, by inactivation of the canonical Wnt/ $\beta$ catenin signaling pathway through CYR61.

Conclusions The newly identified miR-142-5p-CYR61-Wnt/ $\beta$-catenin axis partially illustrates the molecular mechanism of GC recurrence and represents a novel prognosis biomarker for GC.
\end{abstract}

Keywords Gastric cancer $\cdot$ MicroRNA-142-5p $\cdot$ Cyr61 $\cdot$ Prognosis $\cdot$ Metastasis

\section{Introduction}

Gastric cancer (GC) is one of the most common malignancies and a leading cause of cancer-related death worldwide $[1,2]$. The average 5-year survival rate of patients with GC has been reported to be about 25-30\% [3]. As recurrence is a primary cause of GC-related death, it is important to understand the mechanism of recurrent GC.

Electronic supplementary material The online version of this article (https://doi.org/10.1007/s10120-018-0872-4) contains supplementary material, which is available to authorized users.

Rui Xing

xingrui@bjmu.edu.cn

Youyong Lu

youyonglu@hsc.pku.edu.cn

1 Laboratory of Molecular Oncology, Key Laboratory of Carcinogenesis and Translational Research (Ministry of Education), Peking University Cancer Hospital and Institute, 52 Fucheng Road, Haidian District, Beijing 100142, China
In our previous study, we isolated a group of microRNAs (miRNAs) and evaluated their usefulness as prognostic markers for the recurrence of GC through miRNAs microarray and k-top scoring pairs (k-TSP) analysis in 65 patients with GC. We found that miR-142-5p could be a predictive biomarker for GC recurrence $(P=0.059)$; moreover, miR375 and miR-142-5p was a useful signature to predict GC recurrence $(P<0.001)$ [4].

Tumor recurrence is mainly associated with cell proliferation and metastasis [5, 6]. Our previous study showed that miR-375 promoted cell proliferation by directly targeting p53 in GC [7]. However, the function and mechanism of miR-142-5p in GC are still unknown. MiR-142 hairpin gives rise to the "guide strand" miR-142-3p and the "passenger strand" miR-142-5p [8]. Liu et al. pointed that miR-142-5p promoted proliferation and colony formation, but inhibited apoptosis in colorectal cancer (CRC) [9]. Ma et al. reported that miR-142-5p promoted cell proliferation by attenuating TGF- $\beta$ mediated inhibitory effects via targeting SMAD3 [10]. Saito et al. revealed that miR-142-5p suppressed the pro-apoptotic gene TP53INP1 as its target and facilitated the 
pathogenesis of gastric mucosa-associated lymphoid tissue (MALT) lymphoma [11]. Tsang et al. uncovered that miR$142-5 p$, together with miR-142-3p, suppressed hepatocellular carcinoma (HCC) cell migration, probably through several cell motility-associated pathways including those regulating actin cytoskeleton, adherens junctions, and focal adhesion [12]. However, the mechanisms by which miR$142-5 p$ inhibits metastasis were undetermined in GC.

In the present study, we investigated the relationship between miR-142-5p and recurrence in 101 GC tissues and adjacent matched normal tissues, and presented the in vitro and in vivo characteristics of the biological functions and mechanisms of miR-142-5p involved in GC metastasis.

\section{Materials and methods}

\section{Clinical samples and cell lines}

101 pairs of GC tissues and adjacent non-tumor tissues were obtained from Peking University Cancer Hospital from 2002 to 2007. Further, $159 \mathrm{GC}$ tissues and 42 normal gastric tissues were obtained from Hospital 301 from 1996 to 2005. All patients were confirmed to have gastric cancer, and formalin-fixed paraffin-embedded surgical tumor or adjacent non-tumor biopsy tissue samples were collected prior to therapy. Clinicopathological characteristics and follow-up data of patients were collected from medical records, and all patients gave written informed consent for their tissues to be used in research.

Five GC cell lines, BGC823, MGC803, SGC7901, AGS and $\mathrm{N} 87$, were used. All cell lines were cultured in DMEM medium (Gibco BRL, Carlsbad, CA, USA) supplemented with $10 \%$ fetal bovine serum (Gibco BRL) and incubated in a humidified $37^{\circ} \mathrm{C}$ incubator supplemented with $5 \% \mathrm{CO}_{2}$.

\section{Immunohistochemistry}

FFPE sections $(4 \mu \mathrm{m})$ of GC tissues and adjacent nontumor tissues were deparaffinized in xylene and hydrated in a graded alcohol, followed by retrieval in $10 \mathrm{mmol} / \mathrm{l} \mathrm{cit}-$ rate buffer ( $\mathrm{pH}$ 6.0) and endogenous peroxidase treatment with $3 \% \mathrm{H}_{2} \mathrm{O}_{2}$. After incubation with 5\% BSA for $45 \mathrm{~min}$, sections were then incubated with Cyr61 antibody (dilution 1:50, Affbiotech, Cincinnati, USA) at $4{ }^{\circ} \mathrm{C}$ overnight. After incubation with secondary antibody, signal production was conducted using Dako EnVision System (Dako, Glostrup, Denmark). All sections were examined microscopically and scored by two independent professional pathologists from the pathology department of the present hospital who were blinded to the clinical information pertaining to the subjects. The numbers of positive cells in five randomly selected high power fields were counted. Patterns with more than $30 \%$ positive cells were considered positive and less than $30 \%$ positive cells negative.

\section{Transient transfection and establishment of stable transfected cells}

Plasmids were transfected into the cells by using Lipofectamine 2000 (Invitrogen, Carlsbad, CA, USA) according to the manufacturer's protocol. To establish miR-142-5poverexpressing or knockdown stable transfected cells, transiently transfected cells were further seeded in selection medium containing $10 \mu \mathrm{g} / \mathrm{ml}$ of Blasticidin (Invitrogen) for 2 weeks. For Cyr61-overexpressing or knockdown stable transfected cells, $800 \mu \mathrm{g} / \mathrm{ml}$ of neomycin (Invitrogen) was applied for selection. The efficacy of transfection was identified by real-time PCR and western blotting.

\section{Cell proliferation, cell migration and invasion assay}

Cell proliferation was measured using an MTT assay (Promega, Madison, WI, USA) according to the manufacturer's instructions. Absorbance was measured at $490 \mathrm{~nm}$ using a spectrophotometer once a day for four consecutive days. MTT assay data were calculated relative to day 1 .

Cell migration and invasion were measured using a transwell or Matrigel invasion assay (BD Biosciences, Erembodegem, Belgium). Cells in the upper compartment of the chamber were suspended in serum-free medium, and the lower chamber contained medium supplemented with $10 \%$ fetal bovine serum. After $24 \mathrm{~h}$ incubation, cells that passed through the matrigel membrane were fixed and stained with crystal violet and counted in five random microscopic fields.

\section{Immunofluorescence}

Transfected BGC823, SGC7901 and AGS cells were cultured on coverslips, rinsed twice with phosphate-buffered saline (PBS) and fixed with 4\% paraformaldehyde for $15 \mathrm{~min}$. After being permeabilized with $0.5 \%$ Triton X-100 reagent for $20 \mathrm{~min}$, cells were blocked by $5 \%$ bovine serum albumin (BSA) for $1 \mathrm{~h}$ at $37{ }^{\circ} \mathrm{C}$ and labeled with antibody against $\beta$-catenin (1:50) for $2 \mathrm{~h}$ at $37^{\circ} \mathrm{C}$. Cells were washed with PBS three times, 5 min each time, and incubated with FITC-conjugated goat anti-rabbit $\operatorname{IgG}$ for $1 \mathrm{~h}$ at $37^{\circ} \mathrm{C}$. After washing, the samples were mounted and examined under fluorescence microscope.

\section{RNA extraction and quantitative real-time PCR}

Total RNA was extracted from tissues and cells using Trizol reagent (Invitrogen, Carlsbad, CA, USA) according to the manufacturer's instructions. RNA samples with $\mathrm{OD}_{260}$ ' $\mathrm{OD}_{280}$ ratios ranging from 1.9 to 2.0 were considered to be 

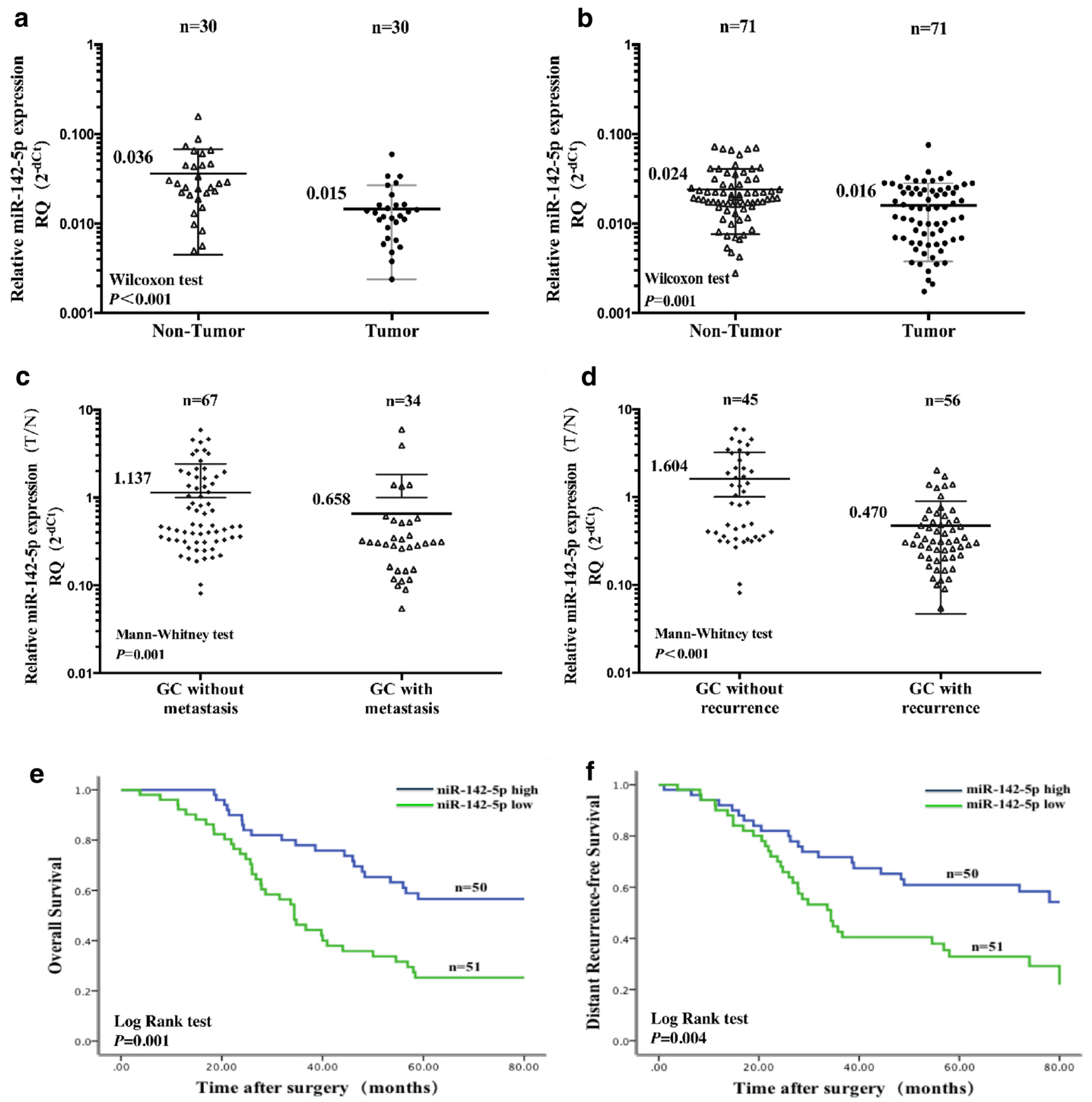

Fig. 1 Low expression of miR-142-5p was in association with metastasis, recurrence and prognosis in patients with GC. a Expression of miR-142-5p in a small discovery cohort $(n=30)$ by qRT-PCR. b Expression of miR-142-5p in a validation cohort $(n=71)$ by qRTPCR. $\mathbf{c}$ The expression of miR-142-5p was lower in GC tissues with metastasis than in GC tissues without recurrence $(P=0.001)$. d The

of good quality. The reverse transcription and quantitative PCR for miR-142-5p and endogenous control RNU6B were performed with GenePharma SYBR Green MicroRNA Assays. Relative expression of miR-142-5p was calculated using a comparative CT method. The reverse transcription

expression of miR-142-5p was lower in GC tissues with recurrence than in GC tissues without recurrence $(P<0.001)$. e, f Kaplan-Meier overall survival curves for GC patients with follow-up information were compared according to miR-142-5p expression level. Patients with lower miR-142-5p expression displayed a longer overall survival and distant recurrence-free survival after surgery

and quantitative PCR for Cyr61 mRNA were conducted using Transgen Reverse Transcription Kit and SYBR Green master mixture with the housekeeping gene $\beta$-actin as an internal control. 

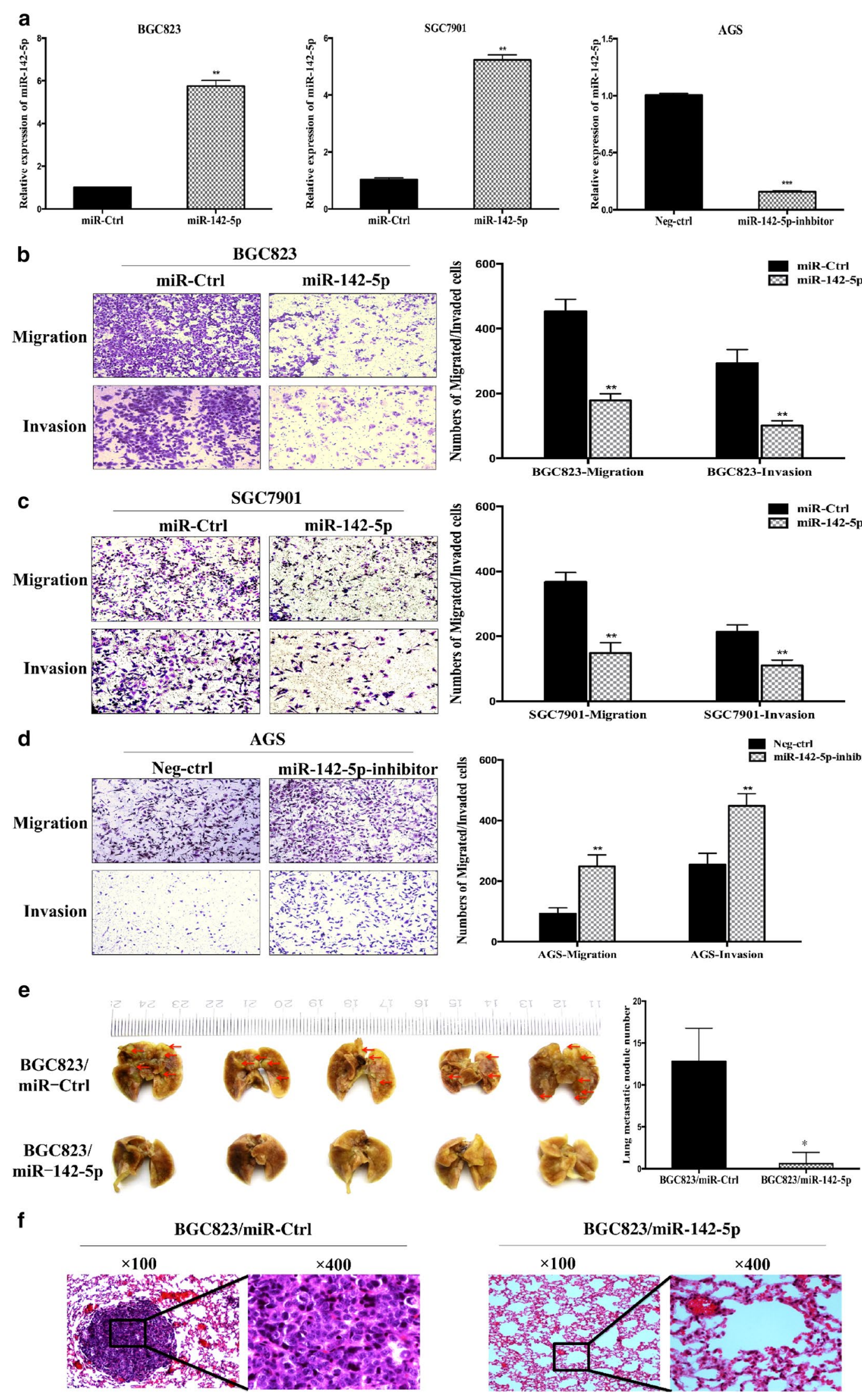
4Fig. 2 Functional studies of miR-142-5p in GC cells in vitro and in vivo. a Left, overexpression of miR-142-5p in BGC823 cells detected by qRT-PCR. Middle, overexpression of miR-142-5p in SGC7901 cells detected by qRT-PCR. Right, knockdown of miR142-5p in AGS cells detected by qRT-PCR. $* * P<0.01$, $* * * P<0.001$. b, c Left, transwell assays were performed without matrigel (upper) and with matrigel (lower). Cell migration and invasion through membrane were remarkably suppressed in miR-142-5p-overexpressing BGC823 and SGC7901 cells, respectively, compared with control cells. Right, the quantification results of migrated cells and invaded cells through membrane were plotted, respectively. Data represented the mean \pm SD of three independent experiments, $* * P<0.01$. d Left, transwell assays were performed without matrigel (upper) and with matrigel (lower). Cell migration and invasion through membrane were remarkably upregulated in miR-142-5p inhibitor-transfected AGS cells compared with control cells, respectively. Right, the quantification results of migrated cells and invaded cells through membrane were plotted, respectively. Data represented the mean \pm SD of three independent experiments, $* * P<0.01$. e The effects of miR$142-5 p$ on the metastasis of BGC823 cells were evaluated in nude mice. Consistent with the transwell assay, metastatic foci to lungs in nude mice were remarkably retarded following miR-142-5p overexpression. ${ }^{*} P<0.05$. f HE staining of lung tissues from mice injected with BGC823 cells stably transfected with miR-Ctrl or miR-142-5p

\section{Western blot}

Total protein was extracted from cell pellets using CytoBuster Protein Extraction Reagent (Merck Millipore, Darmstadt, Germany). Protein measured with 10-30 $\mu \mathrm{g}$ protein from each sample was separated via 6-10\% SDS-PAGE. After protein transfer to nitrocellulose membranes (GE Healthcare, Piscataway, NJ, USA), samples were incubated with primary antibody at $4{ }^{\circ} \mathrm{C}$ overnight and secondary antibody at room temperature for $1 \mathrm{~h}$ (Cyr61: 1:2000, Affbiotech; $\beta$-catenin: 1:2500). Proteins were visualized with ECL Plus Western Blot Detection Reagents (GE Healthcare).

\section{In vivo lung metastasis assay}

BGC823 cells $\left(5 \times 10^{5}\right)$ stably expressing miR-142-5p or control were injected by tail vein into Balb/c nude mice ( $n=5$ mice/group). Mouse lungs were excised and embedded in paraffin for hematoxylin and eosin staining.

\section{Luciferase reporter assay}

The potential miR-142-5p-binding sites in Cyr61 3' untranslated region (3'-UTR) were predicted by miRanda (http:// www.microRNA.org). Sequences containing wild-type or mutant seed region of Cyr61 (Fig. 3b) were synthesized and cloned into luciferase vector (Applied Biosystems). Cells in 12-well plates were transiently co-transfected with miR-142-5p/miR-142-5p-inhibitor or their controls, wild or mutant Cyr61-3'UTR reporter plasmids using Lipofectamine 2000 (Invitrogen). After transfection for $48 \mathrm{~h}$, luciferase activity was measured using a dual-luciferase reporter assay system according to the manufacturer's instructions (Promeaga).

\section{Statistical analysis}

Statistical analyses were performed using GraphPad Prism 6.0 software (GraphPad Software, Inc., La Jolla, USA). Wilcoxon, Mann-Whitney test and $\chi^{2}$ tests were used to analyze miR expression data and its relationship with various clinicopathological factors. Kaplan-Meier analysis and the log-rank test were used for survival analysis. The correlation between two continuous values was analyzed by Spearman's correlation. Univariate and multivariate Cox's proportional hazards models were used to identify independent prognostic factors dictating patient survival. Univariate and multivariate logistic regression models identified independent predictive factors for lymph node metastasis, distant metastasis and recurrence. Data were presented as means $\pm S D$, and all statistical analyses were conducted using the SPSS 19.0 software (SPSS Inc., IL, USA).

\section{Results}

\section{Low expression of miR-142-5p is associated with metastasis in GC}

We detected the expression of miR-142-5p in 30 paired GC and normal tissue samples. The expression of miR-142-5p was significantly downregulated in the GC tissues compared with the matched adjacent normal tissues (median value 0.036 vs. $0.015, P<0.001$ ) (Fig. 1a). This result was further validated in 71 other paired $\mathrm{GC}$ and normal tissue samples (Fig. 1b, $P=0.001$ ). To confirm our results, we also detected the expression of miR-142-5p in the GEO database. The GSE93415 database showed that the expression of miR$142-5 p$ was downregulated in 20 gastric cancer tissues compared to that in adjacent matched normal tissue $(P<0.001)$.

The relationship between the expression of miR-142-5p and the clinicopathological characteristics was further analyzed. Using the median value of miR-142-5p expression (0.403) in the 101 pairs of GC samples as the threshold, the samples were divided into low-expression group and high-expression group (Table S1). Consistent with our previous study, the expression of miR-142-5p in the metastatic (Fig. 1c, $P=0.001$ ) or recurrent GC tissues (Fig. 1d, $P<0.001$ ) was significantly lower, respectively, than in those without metastasis or recurrence. Univariate analyses suggested that the miR-142-5p expression was correlated with distant metastasis (OR 0.343; 95\% CI 0.144-0.817; $P=0.016$ ) and recurrence (OR $0.331 ; 95 \%$ CI 0.146-0.748; $P=0.008)$. Multivariate analysis showed that miR-142-5p expression was not an independent predictor of distant 

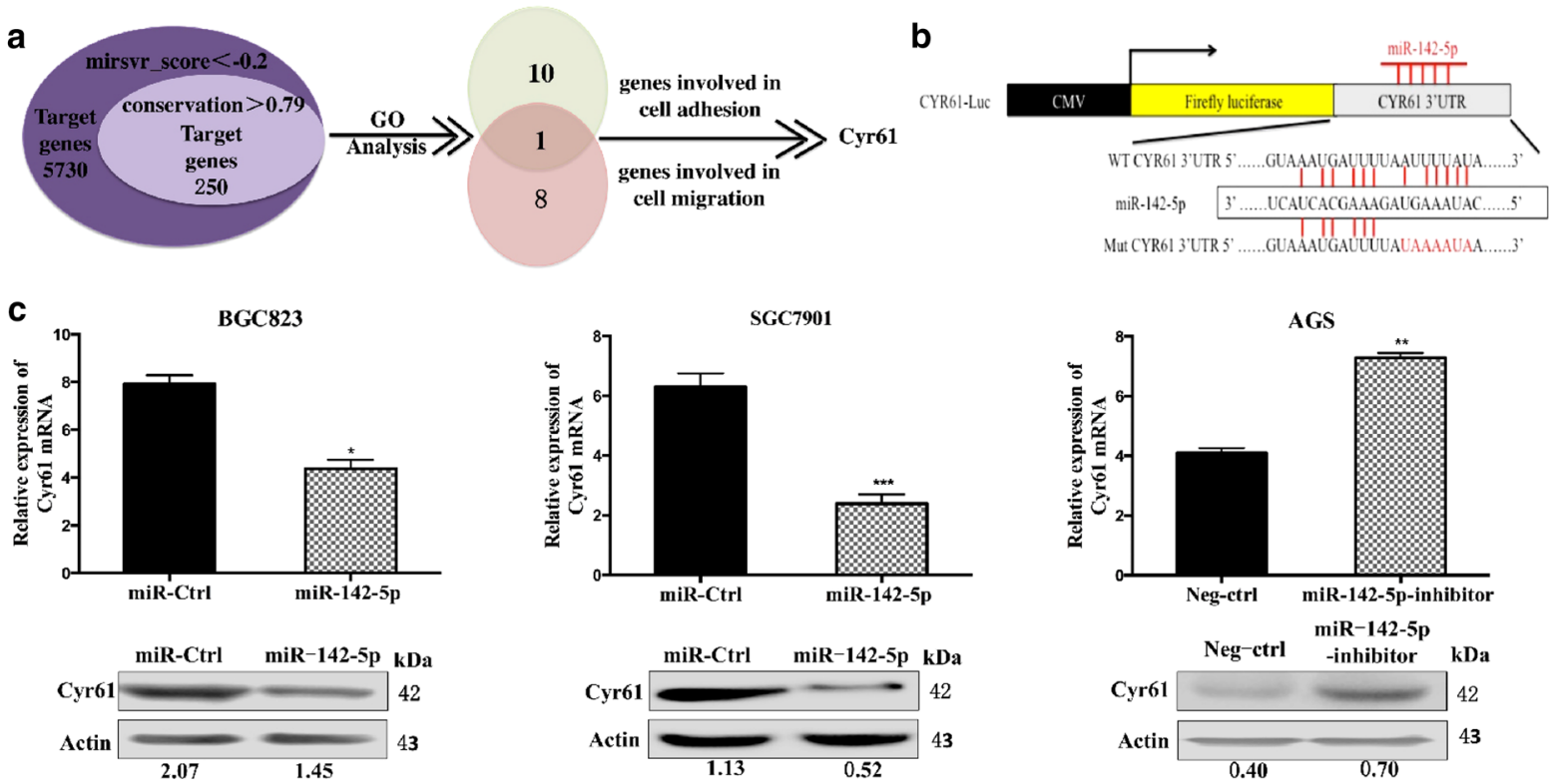

d

BGC823

SGC7901

AGS
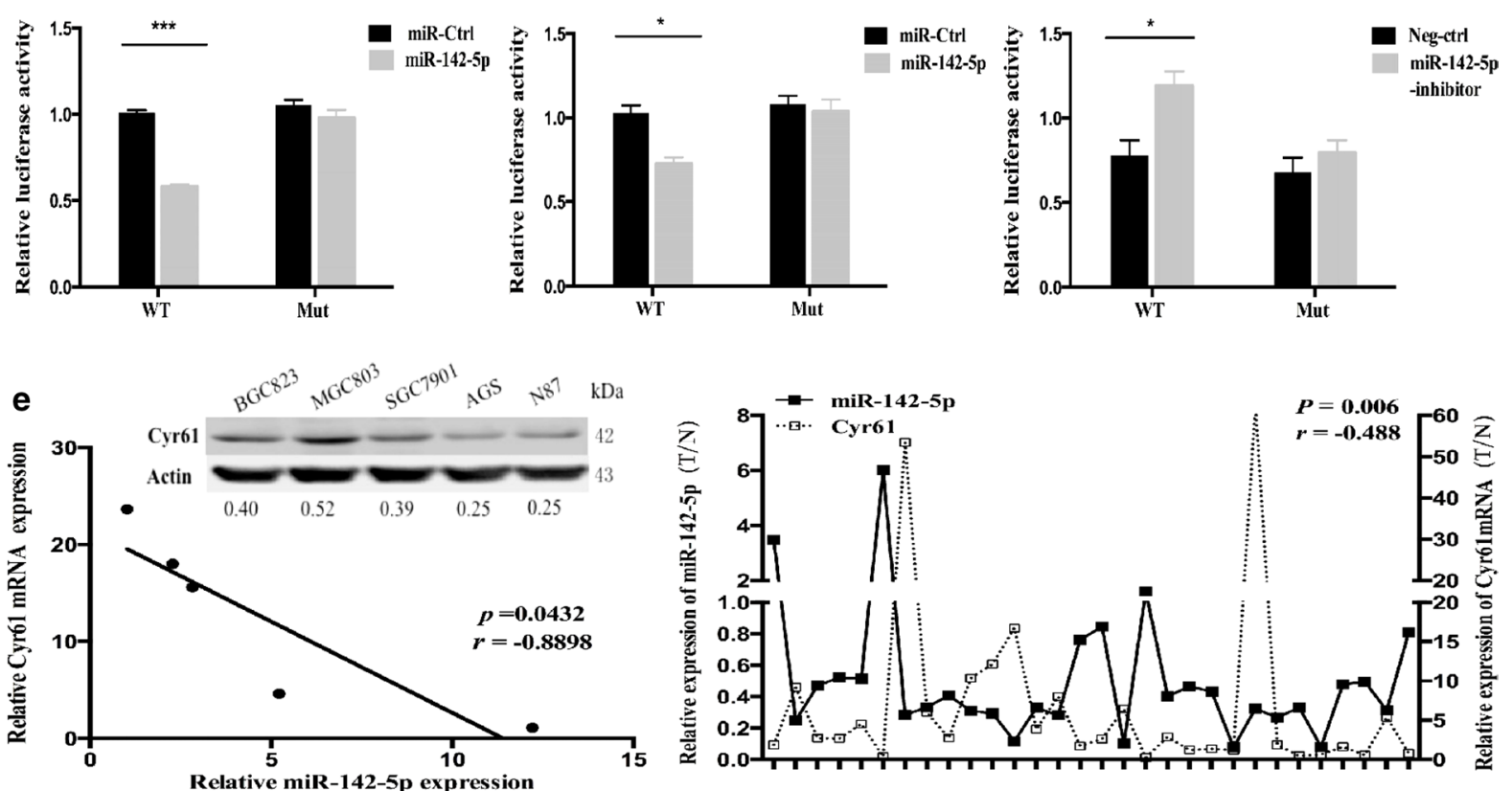

f
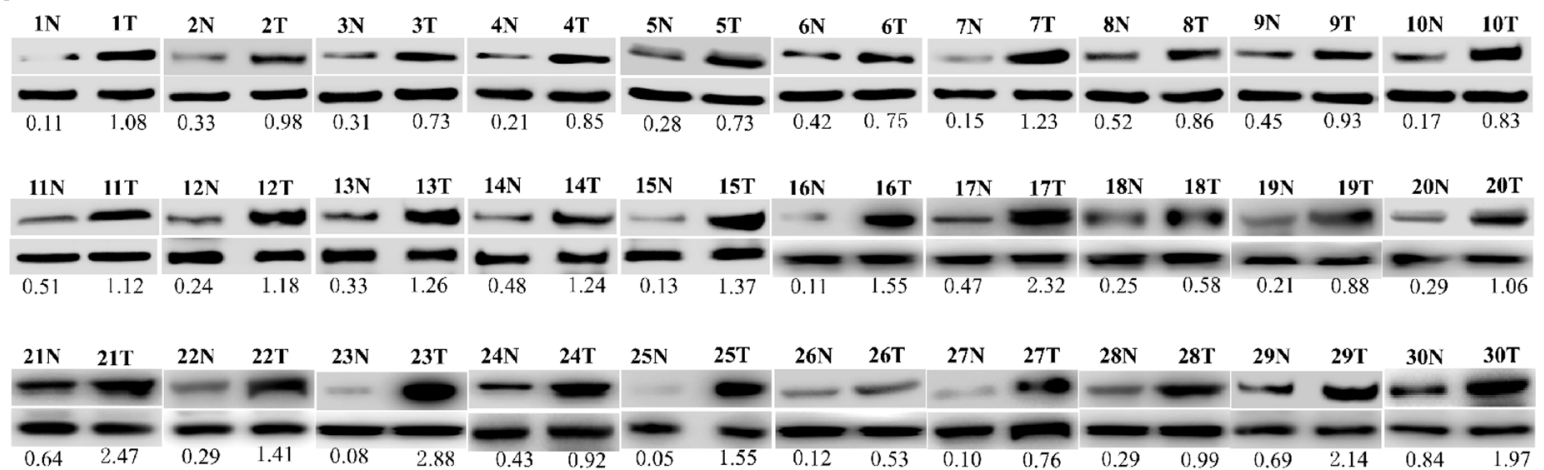
4Fig. 3 CYR61 is a novel target gene of miR-142-5p. a A schematic diagram for screening and identifying the target genes of miR-142-5p involved in metastasis-related pathway. $\mathbf{b}$ The predictive binding sites of miR-142-5p in CYR61 3'UTR and the diagram of CYR61 luciferase reporter plasmids (represented as Cyr61-Luc) with wild-type or mutant CYR61 3'UTR. c Left and middle, qRT-PCR and western blot showed that the expression of $C Y R 61$ was significantly decreased in miR-142-5p-stably transfected BGC823 and SGC7901 cells as compared with control cells. Right, qRT-PCR and western blot showed that the expression of CYR61 was significantly increased in miR142-5p-inhibitor-stably transfected AGS cells as compared with control cells. ${ }^{*} P<0.05$, $* * P<0.01, * * * P<0.001$. d Luciferase reporter assay demonstrated that miR-142-5p inhibited the wild-type, but not the mutant $3^{\prime}$-UTR of CYR61 reporter activities compared with control BGC823 (left), SGC7901 (middle) and AGS cells (right). $* P<0.05$, $* * * P<0.001$. e Left, correlation between miR-142-5p and Cyr61 in GC cell lines. Right, the correlation between miR-142-5p and Cyr61 in 30 GC tissues. f Expression of Cyr61 in 30 GC tissues by WB

metastasis (OR 0.530; 95\% CI 0.192-1.459; $P=0.219$ ), but an independent predictor of recurrence (OR $0.388 ; 95 \%$ CI $0.164-0.918 ; P=0.031$ ) (Table $\mathrm{S} 2$ ). These results suggested that miR-142-5p was involved in metastasis and recurrence of GC.

Kaplan-Meier survival analysis revealed that low miR-142-5p expression was significantly associated with poor overall survival (Fig. 1e, $\log$-rank $P=0.001$ ) and poor distant recurrence-free survival (Fig. 1f, log-rank $P=0.004)$. Univariate analysis revealed that low expression of miR-142-5p was associated with poor prognosis (HR 0.398; 95\% CI 0.232-0.682; $P=0.001$ ). Further multivariate Cox proportional hazard analysis showed that miR-142-5p expression was an independent predictor of prognosis (HR $0.556 ; 95 \%$ CI $0.314-0.983 ; P=0.043$ ) (Table S3).

\section{MiR-142-5p inhibits migration and invasion of GC cells in vitro and in vivo}

To explore the effects of miR-142-5p on GC cells, we ectopically expressed miR-142-5p in BGC823 and SGC7901 cells, which have low endogenous miR-142-5p. As shown in Fig. 2a, the level of miR-142-5p in BGC823 and SGC7901 cells was significantly increased in miR-142-5p overexpressed cells compared with that in control cells. The transwell assay was performed to detect cell migration and invasion in vitro. At $24 \mathrm{~h}$, the number of cells transversing the basement membrane or matrigel in miR-142-5p cells was significantly lower than that in the control cells, either BGC823 cells (Fig. 2b) or SGC7901 cells (Fig. 2c). To further validate the effects of miR-142-5p on migration and invasion, we silenced miR-142-5p expression in AGS cells by stable transfection of miR-142-5p inhibitor. As shown in Fig. 2a, the level of miR-142-5p in the miR-142-5p-inhibitor-stably transfected cells was significantly reduced compared with that in the control cells. The transwell assay showed that the number of cells transversing the basement membrane or matrigel in miR-142-5p-inhibitor-transfected cells was significantly higher than that in the control AGS cells (Fig. 2d). We next performed the lung metastasis analysis in the xenograft nude mouse models to evaluate the metastatic properties of miR142-5p-overexpressing BGC823 cells. As shown in Fig. 2e, $\mathrm{f}$, the cells that metastasized to the lungs were remarkably retarded upon miR-142-5p overexpression. These findings indicated that miR-142-5p markedly attenuated the migration and invasion abilities of GC cells in vitro and in vivo.

Considering that cell migration and invasion capabilities may have resulted from epithelial-mesenchymal transition (EMT), we further examined the expression of EMTassociated markers by western blotting. As shown in Fig. $\mathrm{S} 1$, in AGS cells, knockdown of miR-142-5p significantly decreased E-cadherin expression and increased $\mathrm{N}$-cadherin and vimentin expression compared with control cells. Thus, miR-142-5p may attenuate the migration and invasion abilities of cells by inhibiting EMT in GC cells.

\section{CYR61 is a novel target of miR-142-5p in GC cells}

To explore the potential mechanism involved in the suppressive effects of miR-142-5p on tumor metastasis in GC, we first sought to identify potential miR-142-5p target genes via miRNA target screening using miRanda (mirSVR score $<-0.2$; conservation $>0.79$ ). A total of 250 candidate target genes were acquired, and the Gene Ontology was used to analyze the pathways in which these potential target genes participated. Two metastasis-related terms were gained: cell migration and cell adhesion. CYR61 was the only gene that participated in both pathways (Fig. 3a). The binding sites of miR-142-5p in the 3'UTR of CYR61 are shown in Fig. 3b. qRT-PCR and western blot showed that the expression of CYR61 was significantly decreased in miR-142-5p-stably transfected BGC823 and SGC7901 cells as compared with control cells (Fig. 3c). Consistently, the expression of CYR61 was significantly increased in miR-142-5p-inhibitor-stably transfected AGS cells compared with control cells (Fig. 3c). These results demonstrated that miR-142-5p regulated CYR61 expression at mRNA and protein levels.

To evaluate whether miR-142-5p directly regulated CYR61 expression, dual-luciferase reporter assay was performed. In BGC823 and SGC7901 cells, compared with transfected control cells, ectopically expressed miR-142-5p significantly reduced the luciferase activities of CYR61 containing wild-type 3 '-UTR. Also, miR-142-5p significantly reduced luciferase activities of $C Y R 61$ containing wild-type 3'-UTR compared with mutant 3'-UTR (Fig. 3d). In AGS cells, inhibition of miR-142-5p significantly increased luciferase activities of CYR61 containing wild-type 3'-UTR compared with transfected control cells. Moreover, inhibition of 

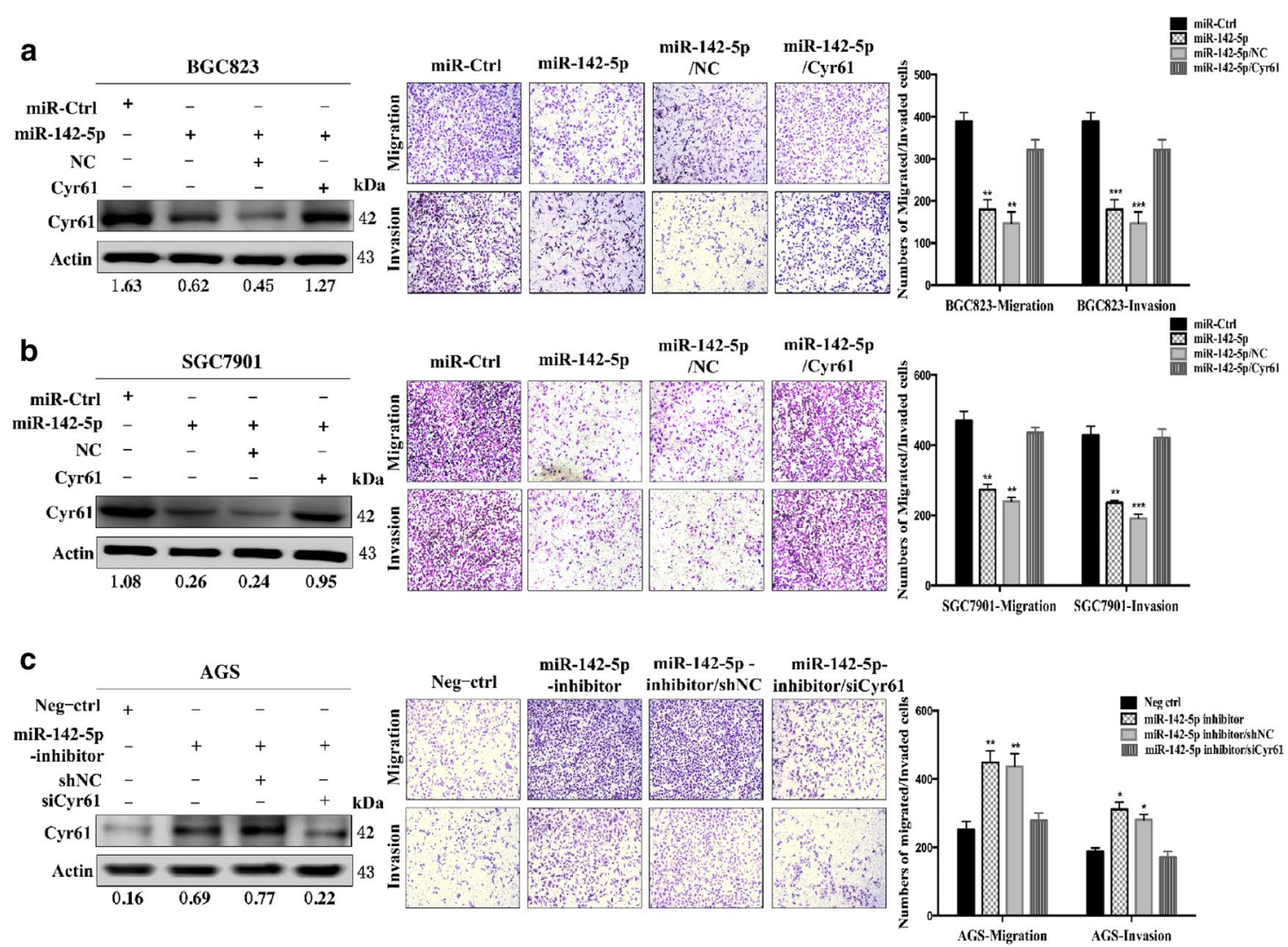

d
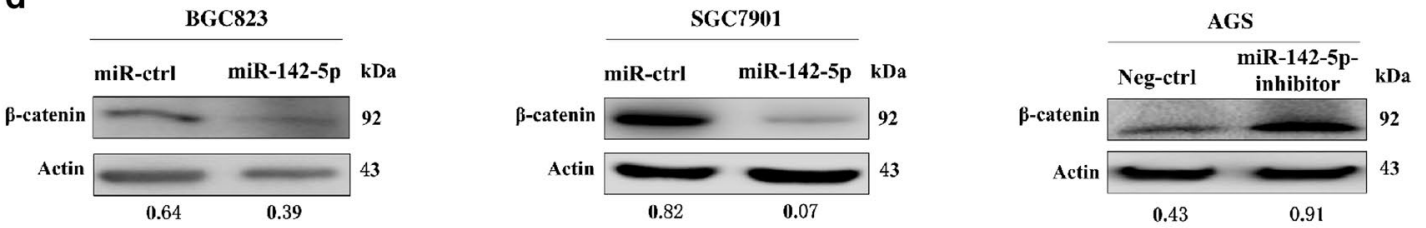

e
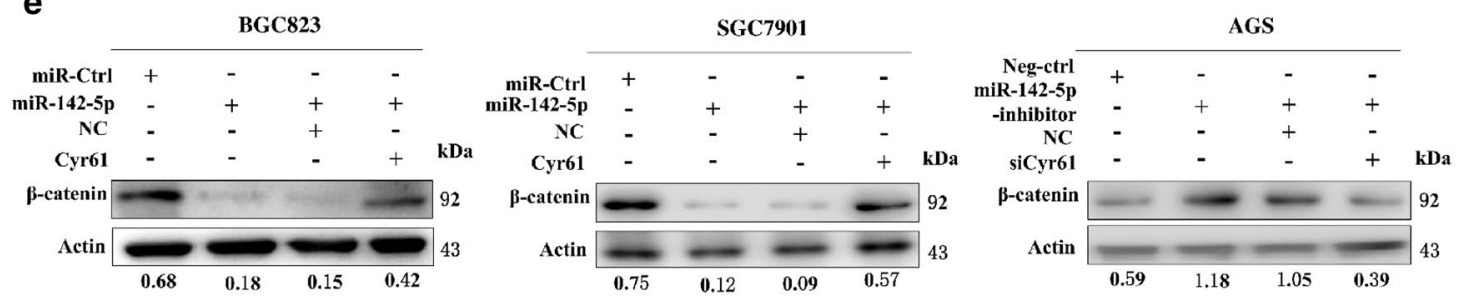

f

BGC823

SGC7901
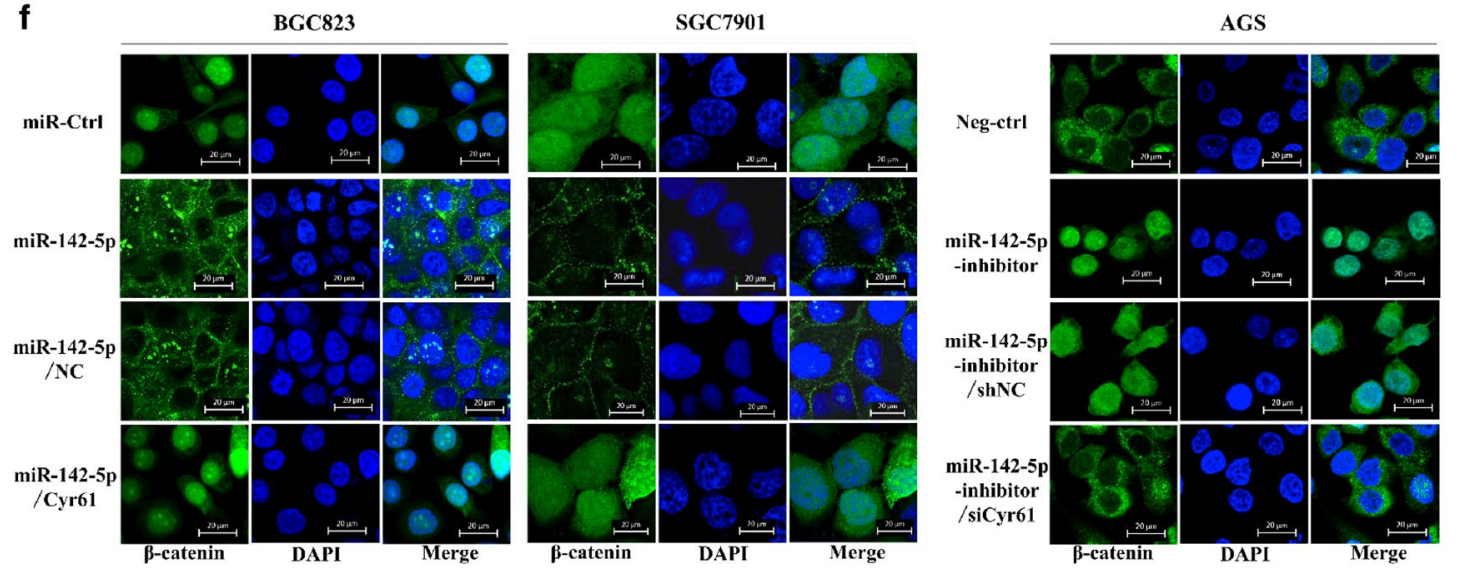
4Fig. 4 Effects of rescued CYR61 expression on functions of miR$142-5 p$ in vitro. a and b Left, western blot detected the CYR61 expression after transfection in the BGC823 (a) and SGC7901 (b) cells stably transfected with miR-142-5p. Middle, transwell assay showed that the migration (upper) and invasion (lower) were significantly restored after the rescued expression of CYR61 in miR142-5p-overexpressing BGC823 (a) and SGC7901 (b) cells. Right, the migrated cells and invaded cells through membrane following rescued expression are counted and plotted, respectively. $* * P<0.01$, $* * * P<0.001$. c Left, western blot detected the CYR61 expression after transfecting shRNA against CYR61 in the AGS cells stably transfected with miR-142-5p-inhibitor. Middle, transwell assay showed that the migration (upper) and invasion (lower) were significantly re-suppressed after the downregulation of CYR61 expression in miR-142-5p-inhibited AGS cells. Right, the migrated cells and invaded cells through membrane following rescued expression were counted and plotted, respectively. The data presented are the mean \pm SD of three independent experiments, $* P<0.05$, $* * P<0.01$. d Left and middle, western blot showed that the expression of $\beta$-catenin was significantly decreased in miR-142-5p stably transfected BGC823 and SGC7901 cells as compared with control cells. Right, western blot showed that the expression of $\beta$-catenin was significantly increased in miR-142-5p-inhibitor stably transfected AGS cells compared with control cells. e Left and middle, western blot showed that the expression of $\beta$-catenin was significantly rescued after transfecting Cyr61 in the BGC823 and SGC7901 cells stably transfected with miR-142-5p. Right, western blot showed that the expression of $\beta$-catenin was significantly re-inhibited after transfecting siCyr61 in the AGS cells stably transfected with miR$142-5$-inhibitor. $\mathbf{f}$ Left and middle, confocal assay showed the location of $\beta$-catenin after transfecting CYR61 in the BGC823 (left) and SGC7901 (middle) cells stably transfected with miR-142-5p. Right, confocal assay showed the location of $\beta$-catenin after transfecting shRNA against Cyr61 in the AGS cells stably transfected with miR142-5p-inhibitor

miR-142-5p significantly increased luciferase activities of CYR61 containing wild-type 3'-UTR compared with mutant 3'-UTR (Fig. 3d). These data suggested that miR-142-5p directly modulated the expression of CYR61 through binding to its $3^{\prime} \mathrm{UTR}$.

The correlation between miR-142-5p and CYR61 expression was further validated in GC cell lines and tissues. The expression of miR-142-5p was inversely correlated with CYR61 mRNA $(P=0.043, r=-0.890)$ and protein in GC cell lines (Fig. 3e). In 30 other pairs of GC tissues and adjacent matched normal tissues, Spearman's correlation analysis demonstrated that CYR61 mRNA and miR-142-5p expressions were inversely related $(P=0.006, r=-0.488$, Fig. 3e). Moreover, the expression of CYR61 was detected in these 30 pairs of GC tissues. As shown in Fig. 3f, consistent with mRNA level, CYR61 protein expression in GC tissues was significantly higher than that in the adjacent matched normal tissues.

\section{MiR-142-5p inhibits migration and invasion via regulating CYR61 in GC cells}

To clarify whether the effect of miR-142-5p on metastasis was mediated through suppressing CYR61 expression, CYR61 without its 3'-UTR was overexpressed in BGC823 and SGC7901 cells stably transfected with miR-142-5p or miRNA control (miR-Ctrl) plasmids (Fig. 4a, b). Migration and invasion assays (Fig. 4a, b) indicated that the number of cells transversing the basement membrane or matrigel in CYR61-transfected miR-142-5p-overexpressing cells was significantly higher than that in the transfected control cells. Consistently, CYR61 was knocked down in AGS cells stably transfected with miR-142-5p-inhibitor or negative control (Neg-Ctrl) (Fig. 4c). Migration and invasion assays (Fig. 4c) indicated that knockdown of CYR61 expression dramatically attenuated the effects induced by miR-142-5p inhibitor.

It is well known that CYR61 activates the canonical Wnt/ $\beta$-catenin pathway. We detected the effects of miR-142-5p on $\mathrm{Wnt} / \beta$-catenin pathway. Ectopic expression of miR-142-5p reduced $\beta$-catenin expression in BGC823 and SGC7901 cells, and induced most of $\beta$-catenin to locate in the cytoplasm and membrane (Fig. 4d, e), while overexpression of CYR61 in cells stably transfected with miR-142-5p increased $\beta$-catenin expression and induced $\beta$-catenin to translocate to the nucleus (Fig. 4f, e). Consistently, inhibition of miR$142-5 p$ expression upregulated $\beta$-catenin expression in AGS cells and induced most of $\beta$-catenin to locate in the nucleus (Fig. 4d, e), while knockdown of CYR61 in cells stably transfected with miR-142-5p-inhibitor reduced $\beta$-catenin and induced $\beta$-catenin to translocate to the cytoplasm (Fig. 4f, e). Collectively, these findings indicated that CYR61 was a critical mediator in the tumor suppressing effect of miR$142-5 p$ in GC.

\section{High expression of CYR61 is associated with metastasis and poor prognosis in GC}

To investigate whether CYR61 expression was linked to the clinicopathological features of GC, we detected the expression of CYR61 in 159 GC tissues and 42 normal gastric tissues by immunohistochemistry. We found that the expression of CYR61 protein in GC tissues was significantly higher than that in normal gastric tissues $(59.75 \%$ vs. $14.29 \%, P<0.001$; Table S4) (Fig. 5a). To confirm our result, we also check the expression of CYR61 in the GEO database. The GSE3438 database showed that the expression of CYR61 was significantly upregulated in 50 gastric cancer tissues compared to that in adjacent matched normal tissue $(P<0.001)$. The relations between the expression of CYR61 and the clinicopathological characteristics in $159 \mathrm{GC}$ tissues were further analyzed (Table S5). Univariate analyses suggested that the CYR61 expression correlated with distant 
a

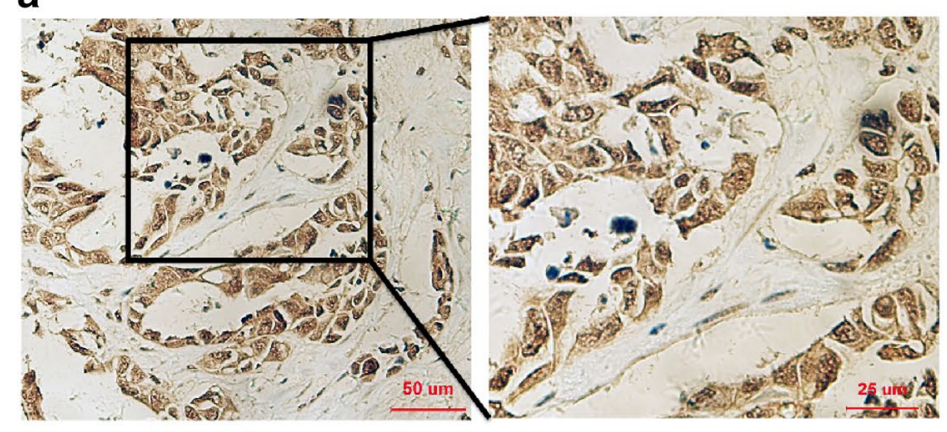

b
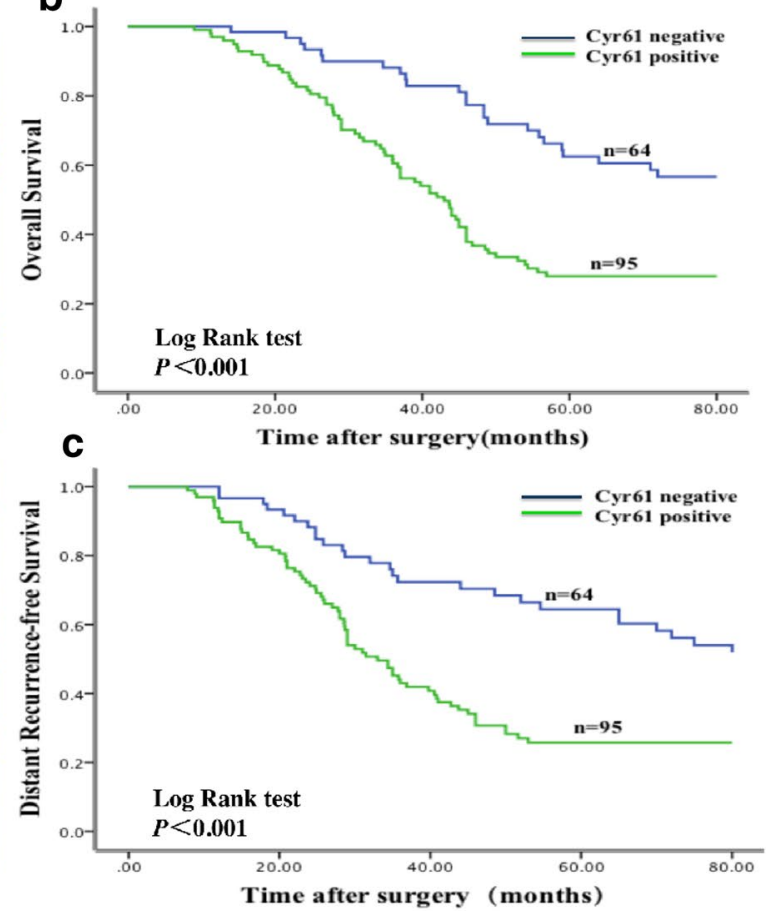

Fig. 5 High expression of CYR61 was associated with prognosis in GC. a The expression of CYR61 in normal gastric tissues (upper) and GC tissues (lower) (left: magnification $\times 200$; right: magnification $\times 400$ ). b, c Kaplan-Meier overall survival curves for GC patients with follow-up information were compared according to the CYR61 expression level. Patients with negative CYR61 expression displayed a longer overall survival and distant recurrence-free survival after surgery metastasis (OR 3.517; 95\% CI 1.599-7.738; $P=0.002)$ and recurrence (OR 3.203; 95\% CI 1.643-6.244; $P=0.001$ ). Multivariate analysis showed that CYR61 expression was an independent predictor of distant metastasis (OR 2.827; 95\% CI 1.253-6.381; $P=0.012$ ) and recurrence (OR 2.550; 95\% CI 1.271-5.115; $P=0.008$ ) (Table S6). Therefore, these results suggested that CYR61 was related to distant metastasis and recurrence.

Kaplan-Meier survival analysis showed that positive CYR61 expression was significantly associated with poor overall survival (Fig. 5b, log-rank $P<0.001$ ) and poor distant recurrence-free survival (Fig. 5c, log-rank $P<0.001$ ). Univariate analysis revealed that positive expression of CYR61 was associated with poor prognosis (HR 2.595; 95\% CI 1.625-4.146; $P<0.001)$. Moreover, multivariate analysis showed that the expression of CYR61 was an independent prognostic factor (HR 1.852; 95\% CI 1.139-3.013; $P=0.013$ ) (Table S7).

\section{Discussion}

We reported previously that low expression of miR-142-5p could predict tumor recurrence; however, the mechanism of miR-142-5p in inhibiting recurrence remained elusive. In the present study, we found that low expression of miR-142-5p was an independent predictor of recurrence and poor prognosis. Decreased expression of miR-142-5p promoted cell migration and invasion through directly targeting CYR61. CYR61 expression could be used as an additional biomarker to identify the GC patients at an increased risk of tumor metastasis. These findings underscore the potentially important role of miR-142-5p downregulation in the development of GC.

Consistent with our previous study [4], the data from the present study further confirmed that the expression of miR$142-5 p$ was significantly downregulated in GC tissues. This is also in line with other reports on various tumors, including HCC and melanoma [12, 13]. Recently, many studies have reported that miRNAs, such as miR-939, miR-206 and miR29c, were associated with recurrence of GC [14-16]. Our multivariate analysis showed that miR-142-5p was an independent predictor of the recurrence and prognosis, implying that miR-142-5p downregulation could specifically predict the most aggressive and fatal types of GC with recurrence.

Liu et al. reported that miR-142-5p could suppress cell growth in lung cancer in vitro [17], and Tsang's group found that miR-142-5p could inhibit hepatocellular carcinoma cell migration in vitro [12]. Our study was the first to provide a novel and comprehensive insight into the functional role of 
miR-142-5p as it related to the recurrence and metastasis of GC. With a series of in vitro and in vivo experiments, we found that miR-142-5p could repress cell proliferation (Fig. S2A), cell migration and invasion in vitro, and inhibit lung metastasis in vivo.

The present study showed that metastasis was closely related to the recurrence of GC. To understand the mechanism of miR-142-5p on recurrence of GC, CYR61 was identified as a direct target of miR-142-5p based on bioinformatics analysis and multiply validated evidences. It is well known that CYR61 is a metastasis-related protein. Our data showed that CYR61 could promote GC cell migration and invasion in vitro (Fig. S2B-D). Overexpressed CYR61 was associated with metastasis in various types of tumors through stimulating cell adhesion and migration [18-21]. In breast cancer, tumor-derived CYR61 was shown to promote protease-activated receptor 1-dependent migration and invasion by inducing MMP-1 expression in adjacent stromal fibroblasts [22]. In melanoma, CYR61 was reported to enhance tumor cell adhesion ability through interaction with heparin and integrin VLA-4 [23]. Besides, we also detected the function of CYR61 in normal gastric epithelium cells. As shown in Fig. S3A-C, overexpression of CYR61 did not affect GES1 cell proliferation, but promoted cells migration and invasion.

Due to the important role of CYR61 in tumor metastasis, the molecules that regulate the expression of CYR61 were identified, such as PDGF, FGF2, IL1 $\beta$ and TGF $\beta$ [21]. For example, Han et al. reported that TGF $\beta 1$ treatment induced CYR61 expression in colon cancer cells [24], Hesler et al. showed that TGF- $\beta$ induced CYR61 expression through canonical TGF- $\beta$-ALK5-Smad2/3 signaling in stromal pancreatic stellate cells [25]. Meanwhile, Ma et al. reported that miR-142-5p regulated TGF $\beta$ pathway by targeting Smad3 in various of cancer cells [10]. Our data showed that miR$142-5 p$ partially inactivated the canonical $\mathrm{Wnt} / \beta$-catenin pathway by targeting CYR61. These evidences suggests that miR-142-5p may be a key regulator linking the TGF $\beta$ and WNT pathway to regulate cell metastasis in cancer.

Canonical Wnt/ $\beta$-catenin signaling pathway favors cell migration and invasion in numerous cancers [26]. CYR61 was reported to activate canonical $\mathrm{Wnt} / \beta$-catenin pathway via stimulating $\beta$-catenin nuclear translocation in glioma and non-small cell lung cancer $[27,28]$. Isobe et al. reported that miR-142-3p activated the canonical Wnt/ $\beta$-catenin signaling by directly targeting adenomatous polyposis coli (APC) [29]. Our results showed that miR-142-5p inhibited $\beta$-catenin expression and nuclear translocation through regulating the expression of CYR61. Moreover, previous studies have indicated that the canonical $\mathrm{Wnt} / \beta$-catenin signaling induced CYR61 expression in pancreatic cancer [28, 30]. It is suggested that there might be a feedback loop between CYR61 and $\beta$-catenin expression.
In summary, our data offered convincing evidences that miR-142-5p-Cyr61-Wnt/ $\beta$-catenin axis partially illustrates the molecular mechanism of GC metastasis and represents a novel potential target for GC treatment.

Acknowledgements This study was supported by National Key Research and Development Program of China (2017YFC1308900), Beijing Municipal Commission of Health and Family Planning Project (PXM2018_026279_000005), National Natural Science Foundation of China (81572346, 81772502), Beijing Natural Science Foundation (7182027), National Bio-Tech 863 Program (No. 2012AA02A203), Beijing Nova Program (Z151100000315069), Beijing Talent Fund, and Beijing Municipal Administration of Hospitals Clinical Medicine Development of Special Funding Support (ZYLX201701).

\section{Compliance with ethical standards}

Ethics approval This study was conducted with the approval of the Institutional Ethical Standards Committee.

Conflict of interest The authors declare that there is no conflict of interest.

Human rights statement and informed consent All procedures followed were in accordance with the ethical standards of the responsible committee on human experimentation (institutional and national) and with the Helsinki Declaration of 1964 and later versions. Informed consent or substitute for it was obtained from all patients for being included in the study.

Animal studies All institutional and national guidelines for the care and use of laboratory animals were followed.

\section{References}

1. Chen W, Zheng R, Baade PD, Zhang S, Zeng H, Bray F, et al. Cancer statistics in China, 2015. CA Cancer JClin. 2016;66:115-32.

2. Torre LA, Bray F, Siegel RL, Ferlay J, Lortet-Tieulent J, Jemal A. Global cancer statistics, 2012. CA Cancer J Clin. 2015;65:87-108.

3. Allemani C, Weir HK, Carreira H, Harewood R, Spika D, Wang XS, et al. Global surveillance of cancer survival 1995-2009: analysis of individual data for $25,676,887$ patients from 279 population-based registries in 67 countries. Lancet. 2015;385:977-1010.

4. Zhang X, Yan Z, Zhang J, Gong L, Li W, Cui J, et al. Combination of hsa-miR-375 and hsa-miR-142-5p as a predictor for recurrence risk in gastric cancer patients following surgical resection. Ann Oncol. 2011;22:2257-66.

5. Ost P, Bossi A, Decaestecker K, De Meerleer G, Giannarini G, Karnes RJ, et al. Metastasis-directed therapy of regional and distant recurrences after curative treatment of prostate cancer: a systematic review of the literature. Eur Urol. 2015;2015:852-63.

6. Paez D, Labonte MJ, Bohanes P, Zhang W, Benhaim L, Ning Y, et al. Cancer dormancy: a model of early dissemination and late cancer recurrence. Clin Cancer Res. 2012;18:645-53.

7. Liu Y, Xing R, Zhang X, Dong W, Zhang J, Yan Z, et al. MiR375 targets the $\mathrm{p} 53$ gene to regulate cellular response to ionizing radiation and etoposide in gastric cancer cells. DNA Repair. 2013;12:741-50.

8. Shrestha A, Mukhametshina RT, Taghizadeh S, Vásquez-Pacheco E, Cabrera-Fuentes H, Rizvanov A, et al. MicroRNA-142 is a 
multifaceted regulator in organogenesis, homeostasis, and disease. Dev Dyn. 2017;246:285-90.

9. Shaojun Liu Z, Xiao F, Ai F, Liu X, Chen K, Cao, et al. MiR142-5p promotes development of colorectal cancer through targeting SDHB and facilitating generation of aerobic glycolysis. Biomed Pharmacother. 2017;92:1119-27.

10. Ma Z, Liu T, Huang W, Liu H, Zhang H-M, Li Q, et al. MicroRNA regulatory pathway analysis identifies miR-142-5p as a negative regulator of TGF- $\beta$ pathway via targeting SMAD3. Oncotarget. 2016;7:71504-13.

11. Saito Y, Suzuki H, Tsugawa H, Imaeda H, Matsuzaki J, Hirata $\mathrm{K}$, et al. Overexpression of miR-142-5p and miR-155 in gastric mucosa-associated lymphoid tissue (MALT) lymphoma resistant to Helicobacter pylori eradication. PLoS One. 2012;7:e47396.

12. Tsang FH, Au SL, Wei L, Fan DN, Lee JM, Wong CC, et al. MicroRNA-142-3p and microRNA-142-5p are downregulated in hepatocellular carcinoma and exhibit synergistic effects on cell motility. Front Med. 2015;9:331-43.

13. Segura MF, Belitskaya-Levy I, Rose AE, Zakrzewski J, Gaziel A, Hanniford D, et al. Melanoma MicroRNA signature predicts post-recurrence survival. Clin Cancer Res. 2010;16:1577-86.

14. Zhang JX, Xu Y, Gao Y, Chen C, Zheng ZS, Yun M, et al. Decreased expression of miR-939 contributes to chemoresistance and metastasis of gastric cancer via dysregulation of SLC34A2 and Raf/MEK/ERK pathway. Mol Cancer. $2017 ; 16: 18$

15. Hou CG, Luo XY, Li G. Diagnostic and prognostic value of serum miR-206 in patients with gastric cancer. Cell Physiol Biochem. 2016;39:1512-20.

16. Han TS, Hur K, Xu G, Choi B, Okugawa Y, Toiyama Y, et al. MicroRNA-29c mediates initiation of gastric carcinogenesis by directly targeting ITGB1. Gut. 2015;64:203-14.

17. Liu X, Sempere LF, Galimberti F, Freemantle SJ, Black C, Dragnev $\mathrm{KH}$, et al. Uncovering growth-suppressive microRNAs in lung cancer. Clin Cancer Res. 2009;15:1177-83.

18. Harris LG, Pannell LK, Singh S, Samant RS, Shevde LA. Increased vascularity and spontaneous metastasis of breast cancer by hedgehog signaling mediated upregulation of cyr61. Oncogene. 2012;31:3370-80.

19. Goodwin CR, Lal B, Zhou X, Ho S, Xia S, Taeger A, et al. Cyr61 mediates hepatocyte growth factor-dependent tumor cell growth, migration, and Akt activation. Cancer Res. 2010;70:2932-41.
20. Monnier Y, Farmer P, Bieler G, Imaizumi N, Sengstag T, Alghisi GC, et al. CYR61 and alphaVbeta5 integrin cooperate to promote invasion and metastasis of tumors growing in preirradiated stroma. Cancer Res. 2008;68:7323-31.

21. Lau LF. CCN1/CYR61: the very model of a modern matricellular protein. Cell Mol Life Sci. 2011;68:3149-63.

22. Nguyen N, Kuliopulos A, Graham RA, Covic L. Tumor-derived Cyr61 promotes stromal matrix metalloproteinase-1 production and protease-activated receptor 1-dependent migration of breast cancer cells. Cancer Res. 2006;66:2658-65.

23. Lin MT, Chang CC, Lin BR, Yang HY, Chu CY, et al. Elevated expression of Cyr61 enhances peritoneal dissemination of gastric cancer cells through integrin alpha2beta1. J Biol Chem. 2007;282:34594-604.

24. Han S, Bui NT, Ho MT, Kim YM, Cho M, Shin DB. Dexamethasone inhibits TGF- $\beta 1$-induced cell migration by regulating the ERK and AKT pathways in human colon cancer cells via CYR61. Cancer Res Treat. 2016;48:1141-53.

25. Hesler RA, Huang JJ, Starr MD, Treboschi VM, Bernanke AG, Nixon $A B$, et al. TGF- $\beta 1$-induced stromal CYR61 promotes resistance to gemcitabine in pancreatic ductal adenocarcinoma through downregulation of the nucleoside transporters hENT1 and hCNT3. Carcinogenesis. 2016;37:1041-51.

26. Pai SG, Carneiro BA, Mota JM, Costa R, Leite CA, BarrosoSousa R, et al. Wnt/ $\beta$-catenin pathway: modulating anticancer immune response. J Hematol Oncol. 2017;10:101.

27. Xiangjun Tong JO'Kelly, Xie D, Mori A, Lemp N, McKenna $\mathrm{R}$, et al. Cyr61 suppresses the growth of non-small-cell lung cancer cells via the $\beta$-catenin-c-myc-p53 pathway. Oncogene. 2004;23:4847-55.

28. Sano M, Wilfredo DRDriscoll, DeJesus-Monge E, Quattrochi B, Appleman VA, et al. Activation of WNT/ $\beta$-catenin signaling enhances pancreatic cancer development and the malignant potential via up-regulation of Cyr61. Neoplasia. 2016;18:785-94.

29. Isobe T, Hisamori S, Hogan DJ, Zabala M, Hendrickson DG, Dalerba $\mathrm{P}$, et al. MiR-142 regulates the tumorigenicity of human breast cancer stem cells through the canonical WNT signaling pathway. Elife. 2014;3:e01977.

30. Weike Si Q, Kang HH, Luu JK, Park Q, Luo W-X, Song, et al. CCN1/Cyr61 is regulated by the canonical Wnt signal and plays an important role in Wnt3A-induced osteoblast differentiation of mesenchymal stem cells. Mol Cell Biol. 2006;26:2955-64. 